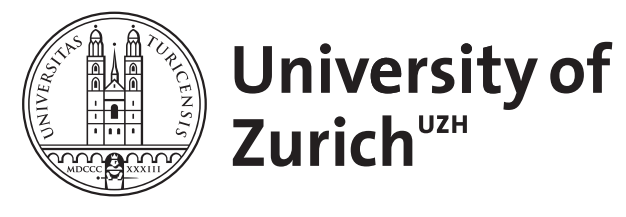

\title{
The state-of-the art in gelotophobia research: A review and some theoretical extensions
}

\author{
Ruch, Willibald ; Hofmann, Jennifer ; Platt, Tracey ; Proyer, Rene T
}

\begin{abstract}
Research on gelotophobia (the fear of being laughed at) has come a long way since the first empirical studies published in 2008. Based on a review of the findings on gelotophobia, its structure, causes and consequences, updates to the model are introduced emphasizing the context of the fear and its dynamic nature. More precisely, external and internal factors are seen to moderate the effects of initial events on gelotophobia, and a spiral nature in the development of the fear is assumed. It is highlighted that gelotophobia needs to be studied in the context of related variables (such as timidity, shame-proneness and social anxiety), and research should focus on the time span in which this fear is most prevalent. The relevance of gelotophobia for humor theory, research and practice is highlighted and new areas of research are introduced. Among the latter the role of gelotophobia at work and in relation to life trajectories is discussed.
\end{abstract}

DOI: https://doi.org/10.1515/humor-2013-0046

Posted at the Zurich Open Repository and Archive, University of Zurich

ZORA URL: https://doi.org/10.5167/uzh-86447

Journal Article

Published Version

Originally published at:

Ruch, Willibald; Hofmann, Jennifer; Platt, Tracey; Proyer, Rene T (2014). The state-of-the art in gelotophobia research: A review and some theoretical extensions. HUMOR: International Journal of Humor Research, 27(1):23-45.

DOI: https://doi.org/10.1515/humor-2013-0046 


\title{
Willibald Ruch*, Jennifer Hofmann, Tracey Platt and René Proyer The state-of-the art in gelotophobia research: A review and some theoretical extensions
}

\begin{abstract}
Research on gelotophobia (the fear of being laughed at) has come a long way since the first empirical studies published in 2008. Based on a review of the findings on gelotophobia, its structure, causes and consequences, updates to the model are introduced emphasizing the context of the fear and its dynamic nature. More precisely, external and internal factors are seen to moderate the effects of initial events on gelotophobia, and a spiral nature in the development of the fear is assumed. It is highlighted that gelotophobia needs to be studied in the context of related variables (such as timidity, shame-proneness and social anxiety), and research should focus on the time span in which this fear is most prevalent. The relevance of gelotophobia for humor theory, research and practice is highlighted and new areas of research are introduced. Among the latter the role of gelotophobia at work and in relation to life trajectories is discussed.
\end{abstract}

Keywords: gelotophobia, humor, laughter, ridicule, review

\footnotetext{
*Corresponding author: Willibald Ruch: Department of Psychology, University of Zurich, Switzerland. E-mail: w.ruch@psychologie.uzh.ch Jennifer Hofmann: Department of Psychology, University of Zurich, Switzerland. E-mail: j.hofmann@psychologie.uzh.ch Tracey Platt: Department of Psychology, University of Zurich, Switzerland. E-mail: t.platt@psychologie.uzh.ch René Proyer: Department of Psychology, University of Zurich, Switzerland. E-mail: r.proyer@psychologie.uzh.ch
}

Not much is known about the downside of humor and laughter. While negative attitudes (e.g., cynicism) and destructive humor behavior (e.g., ridicule) have been looked at, individuals lacking a sense of humor, as well as individuals with even fearful responses towards humor and laughter have received only little attention. How do experiences of being laughed at influence people's feelings, thoughts and behaviors? Furthermore, what personality characteristics serve as protective and promoting factors of being laughed at or bullied?

While being the butt of a joke or being ridiculed is hurtful, and everyone has been laughed at or teased in the past (Proyer et al. 2009), little research on longer lasting effects exists (e.g., Storch et al. 2004). This is surprising, as already Harten- 
berg (1901) wrote that a core component of timid behavior is a disproportional and unjustified fear of ridicule. Unfortunately, Hartenberg's early insights got forgotten and no studies of the fear of ridicule or of being laughed at were conducted subsequently. Had there been, maybe social anxiety disorder nowadays would include a fear of ridicule.

Only recently has empirical research examined whether there is indeed a more enduring fear of being laughed at (Ruch and Proyer 2008a, 2008b), that entails a paranoid sensitivity to the laughter of others, exaggerated responses to being laughed at, and going along with social withdrawal and eventually even the conviction that one is indeed ridiculous (e.g., Ruch 2009; Proyer and Ruch 2010). The first studies emerged from a clinical context (see Titze 2009) before moving to samples of the normal population. Gelotophobia has also found its way into other disciplines (e.g., Daniel 2010; Davies 2009; Lewis 2009; Linge 2013) and has generated much interest from the media and public sphere (e.g., 25500 hits for gelotophobia on google.com in November 2013). Since the first empirical article in 2008, much research has been generated on the topic and a formalized model of putative antecedents and consequences was tested (see Ruch 2004).

\section{Gelotophobia: Conceptual issues and assessment}

After discussing some conceptual issues (definition, measurement, separation from related constructs; social anxiety), a review on knowledge on gelotophobia is given, elaborating on its nature as a shame-bound anxiety, and structured by the proposed model. Furthermore, possible model specifications are discussed and an outlook on what we still need to find out in gelotophobia research is provided.

\subsection{Concept}

Gelotophobia (Greek: gelos = laughter, phobos = fear) has been defined as the fear of being laughed at and appearing ridiculous to social partners (Ruch and Proyer 2008a). While the concept originated in a clinical context, the study of the fear of being laughed at within normal populations led to the postulate that gelotophobia is best conceptualized as a continuum, ranging from no fear to very strong fear (Ruch and Proyer 2008b). It was assumed that individuals at the high end of this continuum fear or anticipate being shamed by the ridicule of others. Consequently, they respond fearfully even to positively motivated, benevolent laughter. 


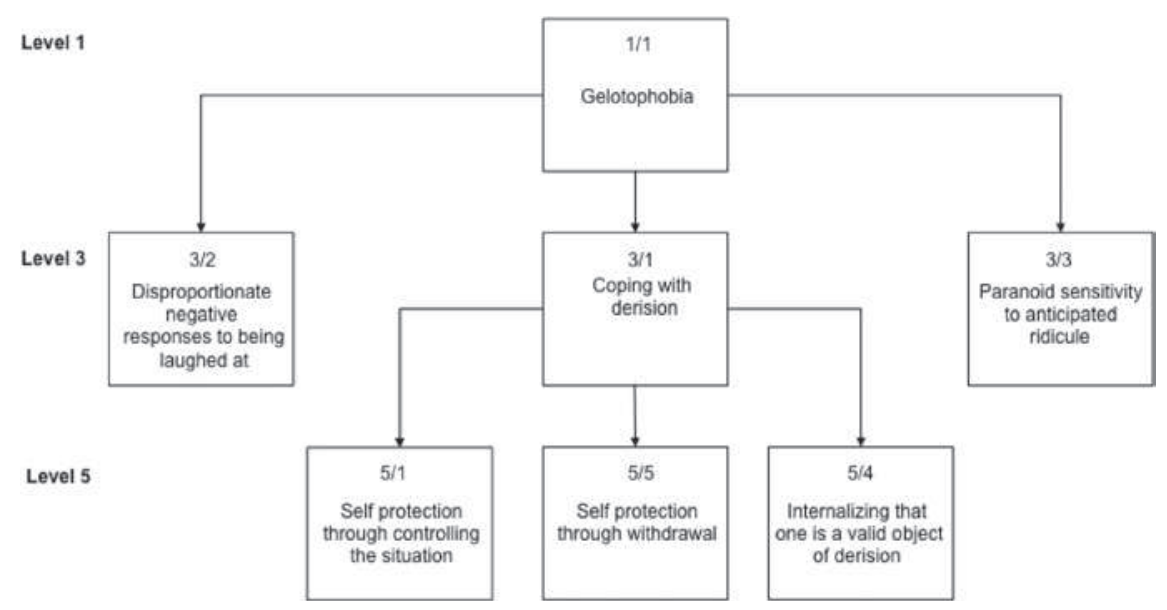

Fig. 1: A hierarchical model of gelotophobia showing a general factor at level 1, three components of negative responses, paranoid sensitivity, and coping at level 3 with the latter decomposing into its elements of control, withdrawal, and internalizing at level 5.

Factor analyses of descriptors of gelotophobia tend to suggest that the concept is uni-dimensional when considering the entire spectrum from no fear to high fear (Carretero-Dios, Ruch et al. 2010; Ruch and Proyer 2008a). However, in the subgroup of gelotophobes (i.e., leaving aside those with no fear or only borderline fear) a hierarchical factor analysis (inspecting all solutions between one and five factors) yielded three positively correlated components, namely coping with derision, disproportionate negative responses to being laughed at, and paranoid sensitivity to anticipated ridicule (Platt et al. 2012). Extracting more than three factors separated coping with derision into components of control, withdrawal, and internalizing (see Figure 1).

This is only a structural model, and future studies will have to investigate whether the factors also play a different temporal role in the genetics of gelotophobia. Strong negative responses to being laughed at might be there at the outset and determine how painful being laughed at is, and the wish to avoid future ridicule. There also might be stages in the coping with derision with attempts at controlling (of oneself and the situation) coming first, then withdrawal, and finally internalizing that one is a ridiculous person. It should be highlighted that separating these components is only fruitful when studying high scorers. Whether gelotophobia is a taxon (Meehl 1992) and qualitatively different from the complement (i.e., the non-fearful) yet needs to be studied. 


\subsection{Assessment}

Gelotophobia can be reliably assessed by the GELOPH $<15>$ (Ruch and Proyer 2008b), a 15-item questionnaire (score range 1.0-4.0), with empirically derived cut-off points allowing for a split between no (1.0-2.5), slight (2.5-3.0), marked (3.0-3.5) and extreme (3.5-4.0) fear of being laughed at (each of these steps equals the length of a standard deviation). The cut-off point was set where individuals agree to half of the gelotophobia items and the distributions of the clinically tested gelotophobes and control group overlapped. The GELOPH<15> has been translated into more than 40 different languages (e.g., Carretero-Dios, Proyer et al. 2010; Chen et al. 2010; Chlopicki et al. 2010; Hrebickova et al. 2009; Kazarian et al. 2009; Samson et al. 2010; Sarid et al. 2011; Stefanenko et al. 2011; Ujlaky et al. 2012) and the comparison of data from different countries allows for describing cross-cultural differences (Chen et al. 2013; Proyer et al. 2009).

The GELOPH $<15>$ items are also used in the PhoPhiKat<45> (Ruch and Proyer 2009a), which additionally measures gelotophilia (i.e., the joy of being laughed at) and katagelasticism (i.e., the joy of laughing at others) in adults and children and adolescents (Proyer, Neukom et al. 2012; Proyer, Meier et al. 2013). Furthermore, a structured interview and a semi-projective tool (Picture GELOPH) are available.

\subsection{Separation from social anxiety, and other concepts}

When a new concept is being introduced it is also important to demonstrate its relative novelty and uniqueness. Research has shown that gelotophobia cannot be fully explained by global personality dimensions, and is also sufficiently different from lower order concepts, such as shame-proneness, timidity, and social anxiety. Regarding the former, gelotophobes turned out to be primarily neurotic introverts, with added minor contributions by paranoid tendencies (Ruch and Proyer 2009b), or low openness (Ruch et al. 2013). However, multiple stepwise regression analyses showed that the sum of predictors (including demographics) explained only between $53 \%$ and $61 \%$ of the variance, suggesting that gelotophobia transcends the personality dimensions used for describing normal adults. As there are hundreds of concepts (e.g., anxiety, depressivity, low self-esteem, etc.) in the introversion neuroticism quadrant of personality, gelotophobia will be related to many if not all of them to a certain extent. Ruch et al. (2013) looked at theoretically relevant concepts and reported that neither lower order traits (e.g., inhibitedness, insecurity), nor a narrow factor of timidity (cf. Hartenberg 1901), nor a combination of narrow traits and introversion and neuroticism 
can fully explain gelotophobia: in each case, about half of the variance is left unexplained.

Likewise, shame-proneness and social anxiety were shown to be sufficiently distinct from gelotophobia. Proyer et al. (2010) found that shame-proneness $(r=.42)$ and the externalization of shameful situations $(r=.24)$ together predict gelotophobia (25\% overlapping variance). While gelotophobes tend to react to a variety of laughter situations with shame (see below), gelotophobia is clearly more than a form of shame-proneness. Furthermore, studies (Carretero-Dios, Proyer et al. 2010; Edwards et al. 2010) showed that gelotophobia could not be fully accounted for by measures of social phobia, and Weiss et al. (2012) found that only $36 \%$ of gelotophobes in a sample of students additionally met the criteria for social phobia (as assessed by a standard clinical interview); i.e., some people feared being laughed at but did not appear as socially anxious. Finally, exploratory and confirmatory factor analyses indicated that gelotophobia forms a distinct factor in joint analyses with measures of fear of negative evaluation and social anxiety (Carretero-Dios, Proyer et al. 2010). So far no study has taken into consideration the heterogeneity of the concepts. For example, Hofmann at al. (2004) discussed subtypes of social anxiety, and Platt et al. (2012) speculated that their three subfactors of gelotophobia (paranoia, excessive responses, and coping) might correlate differently with social anxiety.

Clearly more research is needed here. For example, it needs to be examined, ideally in the same study, whether timid, socially anxious, and shame-prone individuals show the same distortions in relation to laughter (e.g., no discrimination between ridicule and teasing, not being able to identify friendly laughter) than high scorers in the GELOPH $<15>$ do. Likewise, this set of concepts could be used to predict cognitions, emotions, and actions in a variety of situations that are or are not pertinent to ridicule. One might predict, for example, that shame-proneness will predict self-blame, feelings of shame, and behavior inhibition in many settings where self-consciousness is raised, but only gelotophobia will show these effects when the danger of appearing ridiculous is present.

\subsection{Prevalence of the fear of being laughed at}

Two parameters complementing each other may be used in describing the amount of gelotophobia in a group, namely the sample mean in the GELOPH<15> (i.e., average level) and the prevalence rate (i.e., the percentage of individuals exceeding the cut-off scores, representing gelotophobes with a slight, marked, or extreme fear). The statistical mean alone is not representative as it is computed mostly across people without a fear. For example, in Swiss samples typically 
95\% of the participants are in the range from 1 to 2.5 and only $5 \%$ between 2.5 and 4; i.e., people are highly unevenly distributed across the two halves of the scale.

The level of gelotophobia partly varies as a function of variables, such as socio-demographic characteristics, mental health status, well-being, and nationality. No gender differences were found, and studies in the adult population did not show age effects on gelotophobia after age 30. However, being in a relationship goes along with lower scores in gelotophobia compared to being single (Platt and Forabosco 2012; Ruch and Proyer 2008a). It remains unclear whether being in a relationship keeps gelotophobia down, or whether gelotophobes have difficulties forming and maintaining relationships. Moving to remote areas is the utmost form of withdrawing from social groups, and scores should be higher in less populated than in more populated areas. While there is preliminary evidence supporting this (Stefanenko et al. 2011), more research is needed.

Cultural variables play a role, as countries differ in how common ridicule as a form of social correction (Davies 2009) or sheer fun of mockery is. Moreover, factors such as saving face, collectivism, power-distance, etc., will have an impact. A study with 75 nations is underway (see Proyer, Ruch et al. 2009) and the results of some countries were compared (Platt and Forabosco 2012) showing that the 16 (mostly European) countries varied largely, both in terms of mean level gelotophobia score (from 1.43 to 1.92; i.e., one standard deviation) as well as the percentage of gelotophobes (1.6-13.0\%). Interestingly, the scores from several sites within one country are very similar (unpublished findings). Lampert and colleagues (2010) provided evidence for ethnic differences (within United States samples) by showing that individuals with a more interdependent self-construal (Asian Americans) were more likely than those with an independent self-construal (European Americans) to report greater concern over revealing themselves as ridiculous in public.

What groups have high mean levels or prevalence rates? Not surprisingly, a group of clinically diagnosed gelotophobes $(n=99)$ scored highest (appr. 2.99; marking the lower bound of extreme fear) in the GELOPH $<15>$, and $92.9 \%$ of them exceeded the cut-off point (Ruch and Proyer 2008b). Of these 31.3\% were slight, $39.4 \%$ marked, and 22.2\% extreme gelotophobes. Psychiatric samples in Italy (Forabosco et al. 2009) and Russia (Ivanova et al. 2012) yielded higher scores compared to healthy adults, and the number of years spent in psychiatric care correlated positively with gelotophobia in Italy. Weiss et al. (2012) found that gelotophobia partially overlaps with Cluster A personality disorder (mostly with paranoid personality disorder but also with schizoid and schizotypal personality disorder) in gelotophobic students, and also Italian patients with personality disorders or schizophrenic disorders scored higher than normal controls and 
other diagnostic groups (Forabosco et al. 2009). Comorbidity studies are needed: separating the three components of gelotophobia might yield the result that Cluster A personality disorder converges with paranoid sensitivity to anticipated ridicule and social phobia with coping with derision. The overall level of psychopathy is not related to gelotophobia, but, surprisingly, gelotophobia was higher in some subfactors (manipulative and impulsive lifestyle, callous and unemotional) and lower in others (superficial charm, grandiosity; Proyer, Flisch et al. 2012).

Also interesting to study is the autistic spectrum, as those individuals are probably more likely to be ridiculed due to a lack of understanding of social rules, and be more prone to misinterpret laughter due to lack of empathy skills. Indeed, the mean level in gelotophobia in individuals with Asperger's syndrome was 2.46 (Samson et al. 2010) with a prevalence rate of 45\% (27.5\% slight, 10.0\% marked, $7.5 \%$ extreme fear). Bullying victims are higher than those that were not bullied with mean levels of 2.37 (British adults; Platt 2008), 2.27 (Danish children; Führ 2010) and 2.69 (Swiss children; Proyer, Neukom et al. 2012).

What groups are relatively unaffected by gelotophobia? This leads into the field of well-being, flourishing and positive psychology traits. Prevalence levels were lower in "happy" countries (e.g., Denmark, Switzerland) and individual levels were lower when emotional intelligence, life satisfaction, and character strengths (e.g., hope, zest, love, bravery) were high (Papousek et al. 2009; Proyer and Ruch 2009a; Ruch et al. 2010). The understanding of these protective factors might also be necessary for developing both preventive and curative interventions for gelotophobia.

\subsection{Gelotophobia, and humor research and practice: Why bother at all?}

There are a number of reasons why humor research should engage in the study of gelotophobia. First, there is a widespread belief that humor and laughter are good for people - that is, for everyone. The use of humor is promoted in a variety of settings (e.g., advertisements, public speeches, workplaces, hospitals) and even virtual avatars are learning to laugh. Cross-cultural studies show that gelotophobia is universal. Thus, humor professionals need to consider that they also deal with gelotophobes; e.g., gelotophobes are less likely to attend a humor meeting, will avoid going on stage (if requested), will not participate in funny events, will only reluctantly join in laughter, and also will not evaluate the event very positively (if they do not disappear once the event has started). Likewise, in experiments on humor and laughter, gelotophobes will bring noise to the data. 
For example, in fMRI studies on the perception of joyful laughter areas processing shame or fear will light up in some individuals, in addition to, or instead of, the areas processing joy. The notion that it is important to control for gelotophobia in experiments has already entered the research practice (e.g., Fink et al. 2011).

Second, gelotophobia complements the research on humor stimuli and on the relationship between the encoder (e.g., intentions of the sender, his/her personality, details of the situation) and the decoder/receiver. Gelotophobes complicate the process of transmitting humor to an audience as they will wrongly appraise benevolent or neutral laughter as malicious, and are more likely see any more ambiguous but playful form of humor as hostile. Maybe we owe our misanthropic theories of humor (i.e., those that strongly highlight its negative side) to gelotophobes. Reviews of historic views on humor and laughter always mention that they have been condemned during certain times and by certain people (e.g., the misogelos, or "laughter haters"). It might be interesting for the history of humor research (and biographers in general) to see whether such individuals demonstrated signs of distorted views of humor and laughter in their private life. In short: A subsample (between 2 and 20\% across nations) of (in-)voluntary everyday consumers of humor and laughter (private and research participants) but also those that expound theories of humor and laughter (as in the case of the misogelasts, the laughter haters) will be at least slightly biased by gelotophobia.

\section{Gelotophobia research: What is known, what is new?}

In 2004, a model of putative causes and consequences of gelotophobia, as proposed by Titze was established (see Figure 2; Ruch 2004; Titze 2009) with the intention to stimulate initial research and to later revise and update the model if and where needed. Since then, some research has tested selected elements of the model (leaving others untested), and further research has been conducted that is not directly related to the initial model but adds new aspects (the boxes showing additional factors and new lines are dashed).

Figure 2 displays the initial hypothesized causes and consequences of gelotophobia (based on case observations and clinical interviews) supplemented by putatively moderating etiological (personal and contextual) factors and by a shift from linear causality to feedback loops and spiral developments. In the following, a condensed summary of findings will be given that shows which facets 


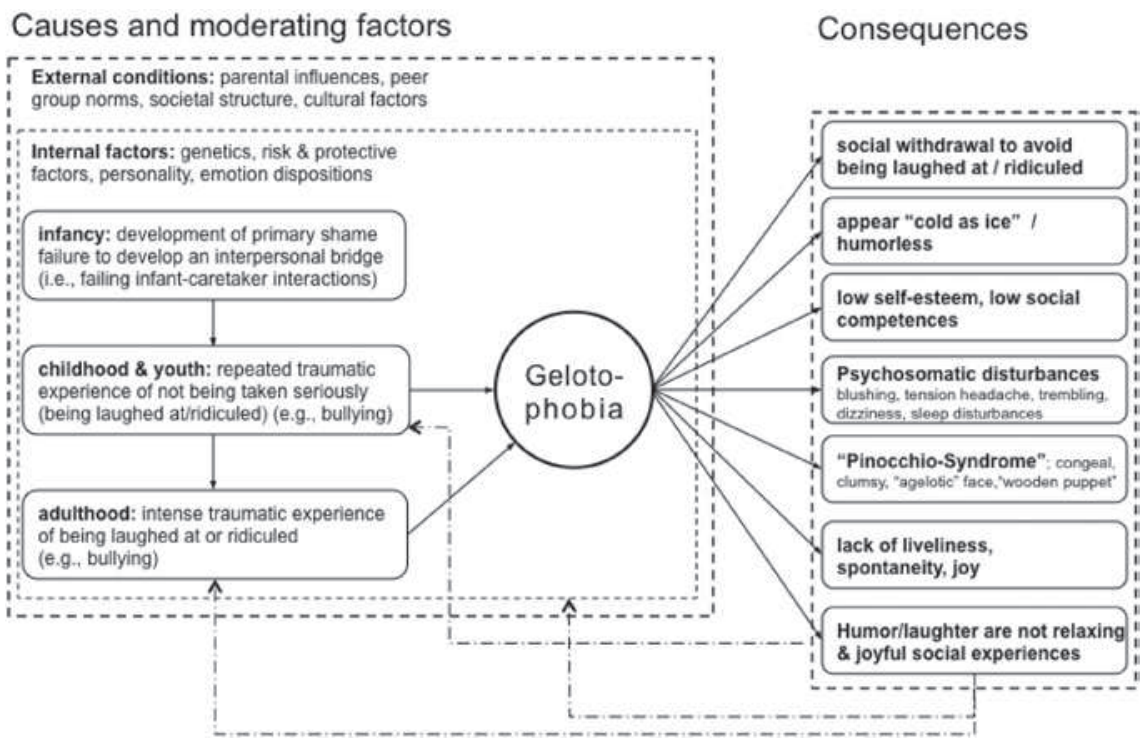

Fig. 2: A revised model of putative causes, moderating factor and consequences of gelotophobia.

of the model, including its additions, have already been tested, and which still lack empirical evidence. It goes without saying that this new model is only an intermediary but not a final step, and it is probably both incomplete and not fully accurate.

\subsection{Gelotophobia, a shame-bound anxiety, and emotions}

Claims that gelotophobia is a shame-bound anxiety stem from early observations of the concept (see Titze 2009). Shame is seen as a type of anxiety, namely shame anxiety, which may range from a slight signal to an overwhelming panic. The gelotophobe fears being exposed, and due to his assumption of ridiculousness will find the situation humiliating. This leads to increased self-observation and self-control to avoid an inappropriate or "funny" impression in others, maybe also to a protective self-presentation style (Renner and Heydasch 2011). They expect rejection by social partners and they scan for clues of possible contempt; especially in the face, as the smiling face might reveal a contemptuous mind. Gelotophobes are convinced that something essential is wrong with them and that they are ridiculous to others; shame-anxiety is driven by feelings of inferiority, insecurity, and self-contempt. 
No study has yet been conducted on the actual feelings at the very moment when gelotophobes get laughed at or anticipate ridicule. As ethical reasons prohibit the activation of this fear, memories offer a substitute, and there are case studies underway in which gelotophobes describe their feelings when they got laughed at the most in their lives (Platt et al. 2012). It is likely that people experience shame but also other negative emotions (anger, disgust) when they got laughed at the most or for the first time. Fear might then come in the anticipation of the reoccurrence of the humiliating event. Studies are needed that explain what leads to the (proper or inaccurate) appraisal that one is currently being ridiculed. This will also require the situational clues that facilitate (e.g., grinning) or impair (e.g., play signal) this appraisal.

There are several studies demonstrating the relevance of three emotions at the trait level: shame, anxiety, and also (low) joy. Compared to the low scorers, the high scorers on the GELOPH<15> experienced shame and fear at higher intensities and at longer duration, and more often during a typical week (Platt and Ruch 2009). Both emotions are also higher during laughter situations and other experimental tasks (Platt 2008; Rawlings et al. 2010). Nearly the obverse applies for joy. Platt and Ruch (2009) showed for German and English gelotophobes that their self-rated most joyful experience in their lives was of a lesser intensity, longer latency, shorter duration, and less strongly facially expressed (Germans only) than the ones reported by non-gelotophobes. However, it is not so much the actual level of shame or joy propensity but the relative dominance of the two (Platt 2008; Platt et al. 2010). More precisely, when the propensity to shame exceeds joy (i.e., in the shame-dominant group) there were $53.33 \%$ gelotophobes compared to $4.50 \%$ gelotophobes in the joy-dominant group. There are several ways how high trait joy might counteract an appraisal of being laughed at. A high disposition to joy is conducive to both state joyfulness and laughter; thus, the maintenance of high levels of state joy and shame-anxiety are incompatible. Laughter is contagious; hearing others laugh is a stimulus for one's own laughter and will be more so among joyful individuals, and one's own laughter will override any impulse for generating shame-anxiety. Furthermore, being prone to laughter oneself will help the person understand that the other person laughing is experiencing joy (not contempt) and this will be antagonistic to suspicious thoughts about the nature of the laughter of others.

Future studies might investigate the nature of the emotions in different stages of the development of gelotophobia. Initial ridicule might induce a variety of negative emotions, but shame will become a dominant one when individuals begin to believe that something is wrong with them. Fear will also get more salient after the initial stage as one anticipated the repetition of humiliation. Contempt for those who do this may raise and sadness that this happens, followed by 
depression once they internalized that they are ridiculous. The presence of a strong habitual inclination to joy or high current joy levels will hinder the process described above.

\subsection{Causes of gelotophobia, moderating factors, and spiral developments}

The new additions of moderating factors of the micro- (e.g., the individual, and his/her family, neighborhood), the meso- (e.g., cities, organizations) and macrolevels (e.g., nation, society) of the system to the model need elaboration. The original model summarized Titze's observations and speculations that during infancy, gelotophobes developed a "primary shame" and fail to develop an interpersonal bridge to their care-takers. This facilitates later events in childhood and adolescence (e.g., repeated traumatic experiences of not being taken seriously, being laughed at and bullied in school) and adulthood (e.g., workplace bullying).

Internal factors (e.g., genetics, risk and protective factors, personality, emotion dispositions) are added to the model to emphasize that the contribution of the parents to the child's gelotophobia does not run through environmental factors only and that the same history of ridicule will yield different outcomes for different people. Parents' and offspring's gelotophobia scores are uncorrelated among children ( $r=.09$; Proyer and Neukom in press) but correlated among adults ( $r=.44$; Proyer, Estoppey and Ruch 2012). Although not tested so far (by twin or adoption studies), this correlation will be partly due to genetic factors and these precede the infant-caretaker situations during which primary shame may develop. As gelotophobic parents more likely will have gelotophobic children the study of how gelotophobic caretakers interact (gaze, facial expression) with their infants or children (see Hoehl and Striano 2008) might emulate the recollection of gelotophobes of their caretakers' behavior.

Furthermore, Figure 2 states that even if the putative causes are valid, they will most likely be facilitated or impaired by factors such as affective dispositions (i.e., propensity to shame, fear, and low joy), lower order personality factors (e.g., inhibitedness, timidity; Ruch et al. 2013), or more global traits (i.e., neuroticism, introversion; Ruch and Proyer 2009b).

Further additions to the model are risk factors for a heightened likelihood of being ridiculed and efficient protective factors. So far no study has identified risk samples of children and youth that are more likely to develop gelotophobia. This may include personal features, such as physical appearance deviating from the statistical norm (e.g., being too tall, obese, skinny, or physically deformed) and 
having disabilities (e.g., stuttering), but also deviating from the norm in behavior, attitudes, communication, or not being knowledgeable about factors like culture and norms (e.g., due to recent migration) (Kohlmann 2013). Certain personality configurations will predict efficient coping with ridicule and serve as protective factors; e.g., people might be proactive and use embarrassing events to make others laugh at them when they want to (e.g., they develop gelotophilia).

Finally, the model now considers external conditions, such as parental influences, peer group norms, societal structure, and cultural factors as protective and facilitating factors in the development of gelotophobia. Proyer Estoppey and Ruch (2012) found punishment and rejection of the child and control, and overprotection to be factors predictive of the development of this fear in a study of 100 families. Both gelotophobes and their parents remembered more punishment (e.g., punishment even for minor things, physical punishment) and higher parental control (e.g., worrying that the child might be harmed, pushing the child to become "the best"). Additionally children (but not parents) also reported less warmth (e.g., not supporting the child, or not showing love). These factors need to be studied in a prospective design as fearful children might trigger protection, and odd behavior might provoke rejection and punishment. Weibel and Proyer (2012) investigated remembered social support by teachers, peers, and family and found that gelotophobia went along with overall lower social support and particularly so with lack of support by peers $(r=.57)$; among those reporting the lowest level of social support 50\% were gelotophobes. Meso-level institutions, such as schools, clubs, the workplace, or communities, differ in the degree to which they actively discourage, tolerate, or enjoy laughing at others. Sports fans commonly mock the enemy team, newspapers ridicule selected targets, talent shows expose the less talented to public ridicule, but there are also school programs or measures in the workplace to prevent bullying. At the macro level factors like economic systems, societal structure, and international relations may be relevant. Davies (2009) proposed two clusters of variables fruitful to study in gelotophobia research, namely variables concerned with laughter as pressure to conform and maintain harmony (shame, face, adherence to etiquette and embarrassment), and the existence and maintenance of hierarchies (status divisions and relations of power). Some countries like mocking foreign cultures more than others.

The most important addition in Figure 2 is dynamism; feedback loops are embedded into the model and change is taken into account. It has been noted that coping with the anticipated ridicule may make people behave oddly, causing them to be perceived as involuntarily funny by others and potentially forming the basis of further ridicule. This is why a path was added linking the consequences with the elicitors in childhood/adolescence and adulthood. Personality traits are 
relatively stable in adulthood reaching a peak in stability at the fifth decade, but they are malleable in the earlier years of life. Neurotic introversion is seen as a moderator (and thus antecedent factor) in Figure 2, but components of both introversion (withdrawal, low joy) and neuroticism (e.g., psychosomatic disturbances, low self esteem) are listed as consequences. Most likely, predispositions will be facilitating the development of the fear, and gelotophobia, in turn, will enhance these factors again. This is why a feedback loop points back to the internal factors. Longitudinal studies investigating the development of gelotophobia are needed (ideally beginning with assessment of infant temperament) that cover the entire time span during which gelotophobia typically is developed. Both measured (Führ 2010; Proyer, Meier et al. 2013; Proyer, Neukom et al. 2012) and recollected (Platt et al. 2010) fear of being laughed at show that this fear peaks in childhood and adolescence, subsequently to decline until 30 and then stay stable across the life span (in cross sectional studies). Adults of different age groups remember the onset of the fear being before the age of 20; a later onset was rare (Platt et al. 2010). Several cohorts can be employed in short-term longitudinal studies in which temperament (and other moderating factors) is repeatedly measured, as are the events of being laughed at, and the current level of gelotophobia, all in a design that allows disentangling causes and consequences of gelotophobia.

Taking aside the additions to the model, what evidence of the validity of the putative causes exists? Platt (2008; Platt et al. 2009) was the first to examine the link between bullying and gelotophobia in adults and found a correlation of $r=.47$. Furthermore, she found that half of the bullying victims were gelotophobes (34.5\% slight, 15.5\% pronounced/extreme fear). Having been bullied was also linked with higher fear of being laughed at among 11-16 year old Danish $(r=.33$; Führ 2010), 13-15 year old Swiss ( $r=.20$ and .17, self- and peer-reports, respectively; Proyer, Meier et al. 2013) and already among 6-9 year old Swiss pupils $(r=.22$ and .16 for self- and teacher-reports, respectively; Proyer, Neukom et al. 2012). Bully victims tend to experience fear rather than joy in teasing situations, and gelotophobia mostly accounts for these effects (Platt et al. 2009)

Gelotophobes did not experience a higher recalled frequency of being ridiculed, but the experiences were of higher intensity, regardless of whether the ridicule took place in the last year (Proyer et al. 2009) or during childhood (Edwards et al. 2010; Ruch et al. 2010; Samson et al. 2011). Interestingly, groups differing in level of gelotophobia (gelotophobes, shame-based neurotics, other patients, normal) also differ in mean level of traumatizing experiences, but their intensity and frequency cannot fully explain the former. While the number of remembered putative reasons correlate highly with gelotophobia among normal subjects ( $r=.51$; Ruch et al. 2010) it can not explain individual differences in 
the level of gelotophobia in groups of diagnosed gelotophobes and Asperger's sufferers (Samson, Huber et al. 2010).

Interviews with gelotophobes of different fear levels (e.g., slight, marked, extreme) will better the understanding of the etiology. More information is needed on what started the fear; e.g., what was the initiating event (one intense ridicule or repeated events), the broader context, the initial reaction and attempts to cope, who was there to help, at what age did, etc. No study examined the nature of the eliciting factors. No study examined whether observing others being ridiculed (rather than being ridiculed oneself) alone is sufficient to develop gelotophobia. The role of the ridiculing person (stranger, friend, family) might matter. We don't know under what circumstances the fear ceases after the age of 30 and when it prevails.

\subsection{Consequences of the fear of being laughed at}

Most notable among the model revisions is the acknowledgement that consequences may also become reasons for being laughed at suggesting a spiral development (e.g., gelotophobes are tense but try to appear relaxed, which creates a ridiculous impression and may lead to further ridicule; acting in a humorless way may lead to being ridiculed, reinforcing the view that humor is a weapon). Also, outcomes may render the moderating factors (e.g., a preexisting tendency to withdraw due to introversion). Hence feedback loops to the internal factors were added. Future changes might involve rearranging the consequences to form homogeneous blocks, and also distal consequences could be added, such as lowered life satisfaction (Proyer, Ruch and Chen 2012), lower cheerfulness (Ruch, Beermann and Proyer 2009) or depressive states. Despite the fact that a more dynamic approach is needed, knowledge has been accumulated that will subsequently be reviewed.

The facet of "humor/laughter are not relaxing and enjoyable social experiences" received support. Live humor exchange in groups has not been studied yet, but correlation between questionnaires showed that gelotophobes are particularly low in socially warm or affiliative humor; i.e., they report that they do not like entertaining others or sharing fun in groups. They also rate sexual and aggressive jokes more aversive than those without a fear (Ruch, Beermann and Proyer 2009; Samson and Meyer 2010).

A well-replicated effect is that for gelotophobes "all laughter is bad laughter." This was found in the nature of emotional responses to teasing and ridicule scenarios involving laughter (Platt 2008), in explaining the affective nature of laughter clips and in a semi-projective test (Ruch, Altfreder and Proyer 2009). In a 
similar vein, hearing laughter does not lift the positive mood, as it would for people without gelotophobia (Platt et al. 2013; Ruch, Altfreder and Proyer 2009). Indeed, gelotophobes show the same emotions in response to playful teasing (which, despite being aggressive humor, is also enjoyable and pro-social) as they and the non-gelotophobes associate with ridicule (Platt 2008), namely shame and fear rather than joy. While no one likes to be the butt of a joke and dislikes the negative emotions (e.g., fear, shame, anger) elicited by ridicule, gelotophobes always feel as they were ridiculed, although the scenarios presented to the participants were preselected by experts to represent playful teasing. It needs to be tested whether gelotophobes are not able to distinguish between teasing and ridicule or whether their emotions are just overriding the correct judgment.

The claim that gelotophobes "appear cold as ice/humorless" yielded partial support. Gelotophobes do not lack humor production ability or wit. While they created equally funny punch lines than individuals with no fear, they rated their wit and sense of humor as being lower (Ruch, Beermann and Proyer 2009). This divergence of self-perception and ability was also found for intelligence and virtuousness (Proyer and Ruch 2009a; Ruch et al. 2009b). No rating study of gelotophobes appearing cold as ice has been carried out yet, neither from clips nor in face-to-face interactions. A good test of the hypothesis would not only use ratings of whether the person shows affection or feelings generally but would also use more interpersonal attributes to see whether the differences are restricted to appearing distant and cold.

Evidence that gelotophobes "lack liveliness and spontaneity" is still scarce but the claim for "lacking joy" is well supported. As the former are facets of extraversion they are partially covered by the finding that gelotophobes are introverts (Ruch et al. 2013). There is ample evidence that gelotophobes have deficits in perceiving, responding to and generating joy. Gelotophobes do not perceive the positive affect in laughter and playful situations, and positive emotions are not contagious for them (Papousek et al. 2009; Ruch, Altfreder and Proyer 2009). Further evidence that gelotophobes do not seem to benefit from joy inducing stimuli comes from experiments of facial responses when recalling enjoyable emotions (Platt et al. 2013). Usually humans smile when they remember a joyful event. During interviews, when describing feelings to scenarios prototypical for 16 enjoyable emotions, gelotophobes did not only show fewer genuine enjoyment smiles, their smiles were also less intense and less frequent in the enjoyable emotions that went along with laughter, e.g. amusement, schadenfreude, and relief (Platt et al. 2013). Hofmann et al. (2013) attempted to verify that gelotophobes have a specific interpreting bias, namely to distrust facial expressions depicting smiling and laughter but found, more severely, that gelotophobes seem to 
confuse genuine joy with contempt in both decoding and encoding of emotions. This tendency complicates social interaction by fostering misunderstandings, and may also serve as a maintaining factor of gelotophobia.

The putative consequence of "social withdrawal to avoid being laughed at/ ridiculed" assumes a special role. First, social detachment as part of neurotic introversion might exist before people develop gelotophobia and thus form an antecedent condition (see also Kazarian et al. 2009). Second, withdrawing from social situations in order to avoid being ridiculed is one component of the coping with derision factor (see Platt et al. 2012); i.e., it is part of the definition of gelotophobia. We have kept these items in the GELOPH $<15>$, however, as they show how strongly gelotophobia is developed; i.e., how far coping developed from control, to withdrawal and internalizing ridiculousness.

With respect to "low self-esteem and low social competences", it is known that gelotophobes feel relatively weak in regulating their emotions, and experts considered the attempts they typically make to manage their emotions as inefficient. Further, gelotophobes reported having a strong tendency to control their emotion expression. One study revealed differences in the use of intrapersonal emotion-related skills, but provided no evidence that gelotophobia may relate to deficits in interpersonal skills (Papousek et al. 2009).

Regarding the psychosomatic disturbances, unpublished data suggest more complaints (e.g., blushing, trembling, dizziness) but psycho-physiological recordings are missing. The first evidence for a "Pinocchio Syndrome," emerged from Titze's observations of patients. Platt (2011) speculated that faked smiles (compensating for the lack of emotional smiles in interaction) might be one element in the Pinocchio Syndrome, as its cramp-like appearance is perceived as odd and the basis for further ridicule.

The picture drawn of gelotophobes appears one-sided and lacking valued characteristics. This is not entirely the case, as the character strengths of modesty and prudence are more pronounced among gelotophobes in both self- and peerreports (Proyer and Ruch 2009a), and Radomska and Tomczak (2010) showed that gelotophobes do not oversell themselves.

\section{Outlook}

After five years of empirical research on the fear of being laughed at, we can reliably measure gelotophobia all over the globe, not only among adults, but also among children and adolescents. The revision and refinement of the model requires testing of new hypotheses and also the review of the findings highlighted where further studies are needed. 
One of the prime goals of future research is the inquiry into the utility of the concept in a broader variety of life settings where gelotophobia plays a role but has not yet been studied. Under-studied domains are schools, sports, leisure time, youth clubs, as well as the relationship to peers, teachers, siblings, and parents. The fear of being laughed was most pronounced during the teenage years. Developmental changes dealing with puberty occur and some of them will be possible causes for anticipated ridicule. Here, gelotophobia might play a moderating role. Also, acts of retaliation in response to ridicule and being laughed at (at the extreme in the form of school shootings) may be related to gelotophobia. At the same time, gelotophilia and katagelasticism will be relevant as well in a more successful coping with potentially shame-inducing factors or being the source of ridicule, respectively. While gelotophobia decreases after the teenage years it is still of interest to study the more extreme forms of gelotophobia and relate it in the context of models of personality pathology in both children and adolescents.

The study among adults should be expanded to life domains, such as work, family (partnership, marriage, parenting), social and community life in institutions (citizenship, friendship), health, leisure time (sports, cultural activities), etc. Regarding work, gelotophobes may avoid certain types of jobs (where one is likely to get laughed at, such as performing arts) but seek others. Their sensitivity to ridicule but also their peculiar behavior will make them believe to be the target of bullying at the workplace or actually be it, respectively. Unemployment is a shame-inducing life event, and seeking reemployment might be differently difficult to those with and without a fear of being laughed at.

Following the footsteps of Platt and Ruch (2010) gelotophobia may be studied in old age, where vulnerability is high. Problems of a different kind emerge, and people need to ask for help but don't, as they feel ashamed about it and fear being ridiculed. Indeed it was found that people who generally fear being laughed at (but who also ridicule others) expect to act gelotophobic when facing age-related problems (Platt and Ruch 2010; Platt et al. 2010).

The first evidence on the causes and consequences of gelotophobia has been substantiated by cross-sectional research and now it is time to conduct longitudinal studies, at best across the entire life span. Some of this research will be done in a project entitled "LIVES - Overcoming Vulnerability: Life Course Perspectives", which analyzes the burdensome effects of post-industrial economies and societies on the development of vulnerability in terms of social exclusion or precariousness. It also includes longitudinal analysis to examine the impact of socio-structural and personal resources on overcoming vulnerability.

Evaluating a training program that is informed by research findings and is aimed at permanently reducing or eliminating the fear should be the utmost goal. 
Such training will need to address a variety of cognitive, affective etc. layers. As gelotophobes are not generally anhedonic, but only dislike emotions including laughter their well-being may be fostered by utilizing positive emotions untypical for laughter as a start for an upward spiral. Training also needs to highlight the meaning of play signals, and provide help to discriminate between serious ridicule and playful teasing, train interpersonal skills and establish rules for the interaction with partner, family, and peers to minimize misunderstanding. A comparison of high and low prevalence countries may be undertaken, that considers factors like societal acceptance (or appreciation) of ridicule in public and private life, amount of benevolent humor vs. mockery in the media, implementation of anti-bullying programs in schools and other institutions, role of the press, number of "outsiders" in the society, presence and acceptance of models of respectful interpersonal conduct, etc. Thus, preventive and curative factors do require attention at the micro-, meso-, and macro-levels.

We have made progress but are still left with puzzles that do not make sense. For example, within Europe, Britain - whose population is commonly perceived as having a "great sense of humor" - is also (together with Romania) the country with the highest prevalence of gelotophobia (and numerically highest among the extremes; Platt and Forabosco 2012). Likewise, it seems so easy to distinguish between ridicule and teasing when reading a scenario, but apparently very difficult when one is in such a situation. Is it a cognitive ability, or an emotional habit? Further research into gelotophobia is requested.

\section{References}

Carretero-Dios, Hugo, René T. Proyer, Willibald Ruch \& Victor J. Rubio. 2010. The Spanish version of the GELOPH<15>: Properties of a questionnaire for the assessment of the fear of being laughed at. International Journal of Clinical and Health Psychology 10. 345-357.

Carretero-Dios, Hugo, Willibald Ruch, Diana Agudelo, Tracey Platt \& René T. Proyer. 2010. Fear of being laughed at and social anxiety: A preliminary psychometric study. Psychological Test and Assessment Modeling 52. 108-124.

Chen, Guo-Hai, Chao-Chih Liao, René T. Proyer \& Willibald Ruch. 2010. Gelotophobia in China: One the assessment of the fear of being laughed at. China Journal of Health Psychology 18. 887-889.

Chen, Hsueh-Chih, Yu-Chen Chan, Willibald Ruch \& René T. Proyer. 2013. Being laughed at and laughing at others in Taiwan and Switzerland: A cross-cultural perspective. In J. R. M. Davis \& J. V. Chey (Eds.), Modern and contemporary approaches to humor in China (Vol. 2, pp. 215-229). Hong Kong: Hong Kong University Press.

Chlopicki, Wladyslaw, Anna Radomska, René T. Proyer \& Willibald Ruch. 2010. The assessment of the fear of being laughed at in Poland: Translation and first evaluation of the Polish GELOPH<15>. Polish Psychological Bulletin 41. 172-181. 
Daniel, Gwyneth. 2010. Utmost extrication: Why Henry Hellyer shot himself. Wynyard, Australia: Willows Books.

Davies, Christie. 2009. Humor theory and the fear of being laughed at. Humor: International Journal of Humor Research 22(1-2). 49-62.

Edwards, Kim, Rod A. Martin \& David J. A. Dozois. 2010. The fear of being laughed at, social anxiety, and memories of being teased during childhood. Psychological Test and Assessment Modeling 52. 94-107.

Fink, Andreas, Daniela Schwab \& Ilona Papousek. 2011. Sensitivity of EEG upper alpha activity to cognitive and affective creativity interventions. International Journal of Psychophysiology 82. 233-239.

Forabosco, Giovannantonio, Willibald Ruch \& Pietro Nucera. 2009. The fear of being laughed at among psychiatric patients. Humor: International Journal of Humor Research 22(1-2). 233-251.

Führ, Martin. 2010. The applicability of the GELOPH<15> in children and adolescents: First evaluation in a large sample of Danish pupils. Psychological Test and Assessment Modeling 52. 60-76.

Hartenberg, Paul. 1901. Les timides et la timidité [The shy and shyness]. Paris, France: Félix Alcan.

Hofmann, Jennifer, Tracey Platt, Willibald Ruch \& René T. Proyer. 2013. Individual differences in the decoding and responding to joy and contempt. Manuscript under review.

Hofmann, Stefan G., Nina Heinrichs \& David A. Moscovitch. 2004. The nature and expression of social phobia: Toward a new classification. Clinical Psychology Review 24. 769-797.

Hoehl, Stefanie \& Tricia Striano. 2008. Neural processing of eye gaze and threat-related emotional facial expressions in infancy. Child Development 79. 1752-1760.

Hrebickova, Martina, Emilia Fickova, Mirka Klementova, Willibald Ruch \& René T. Proyer. 2009. The fear of being laughed at: Czech and Slovak version of a questionnaire for gelotophobia. Ceskoslovenska Psychologie 5. 468-479.

Ivanova, Alyona, Ekaterina Stephanenko, Stefanenko Enikopolov, René T. Proyer \& Willibald Ruch. 2012. The fear of being laughed at in healthy people and psychiatry patients. Assessing gelotophobia in Russia. Bridging Eastern and Western Psychiatry 8. 10-17.

Kazarian, Shahe, Willibald Ruch \& René T. Proyer. 2009. Gelotophobia in the Lebanon: The Arabic version of a questionnaire for the subjective assessment of the fear of being laughed at. Arab Journal of Psychiatry 20. 42-56.

Kohlmann, Carl-Walter. 2013. Overweight and the experience of teasing and ridicule: Associations with gelotophobia. Paper presented at the 13th Congress of the Swiss Psychological Society, Basel, Switzerland.

Lampert, Martin D., Kate L. Isaacson \& Jim Lyttle. 2010. Cross-cultural variation in gelotophobia within the United States. Psychological Test and Assessment Modeling 52. 202-216.

Lewis, Paul. 2009. Partisan gelotophobia and preemptive humor strategies. Paper presented at the 21st Annual Conference of the ISHS, Long Beach, CA.

Linge, Lotta. 2013. Joyful and serious intentions in the work of hospital clowns: A meta-analysis based on a 7-year research project conducted in three parts. Journal of Qualitative Studies on Health and Well-being 8.

Liu, Cheng-Hong. 2012. Valuable but threatening: The reduced effect of incremental theory on challenge-confronting tendencies for students who fear being laughed at. Learning and Individual Differences 22. 839-843. 
Meehl, Paul E. 1992. Factors and taxa, traits and types, differences of degree and differences in kind. Journal of Personality 60. 117-174.

Papousek, Ilona, Willibald Ruch, Heribert Freudenthaler, Eva Kogler, Brigitte Lang \& Guenter Schulter. 2009. Gelotophobia, emotion-related skills and responses to the affective states of others. Personality and Individual Differences 47. 58-63.

Platt, Tracey. 2008. Emotional responses to ridicule and teasing: Should gelotophobes react differently? Humor: International Journal of Humor Research 21(2). 105-128.

Platt, Tracey. 2011. Interview with a gelotophobe. Individual Differences in enjoyment signal responses to humorous stimuli: A FACS study. Paper presented at the 23 rd Conference of the ISHS, July 5-9, Boston University, MA.

Platt, Tracey \& Giovannantonio Forabosco. 2012. Gelotophobia: The fear of being laughed at. In P. Gremigni (ed.), Humor and health promotion, 229-253. New York, NY: Nova Science.

Platt, Tracey, Jennifer Hofmann, Willibald Ruch \& René T. Proyer. 2013. Duchenne display responses towards sixteen enjoyable emotions: Individual differences between no and fear of being laughed at. Motivation and Emotion 37. 776-786.

Platt, Tracey, René T. Proyer \& Willibald Ruch. 2009. Gelotophobia and bullying: The assessment of the fear of being laughed at and its application among bullying victims. Psychology Science Quarterly 51. 135-147.

Platt, Tracey \& Willibald Ruch. 2009. The emotions of gelotophobes: Shameful, fearful and joyless? Humor: International Journal of Humor Research 22(1-2). 91-110.

Platt, Tracey \& Willibald Ruch. 2010. Gelotophobia and age: Do disposition towards ridicule and being laughed at predict coping with age-related vulnerabilities? Psychological Test and Assessment Modeling 52. 231-244.

Platt, Tracey, Willibald Ruch, Jennifer Hofmann \& René T. Proyer. 2012. Extreme fear of being laughed at: Components of gelotophobia. Israeli Journal of Humor Research: An International Journal 1. 86-106.

Platt, Tracey, Willibald Ruch \& René T. Proyer. 2010. A lifetime of fear of being laughed at: An aged perspective. Zeitschrift für Gerontologie und Geriatrie 43. 36-41.

Proyer, René T., Stephanie Estoppey \& Willibald Ruch. 2012. An initial study on how families deal with ridicule and being laughed at: Parenting styles and parent-child-relations with respect to gelotophobia, gelotophilia, and katagelasticism. Journal of Adult Development 19. 228-237.

Proyer, René T., Rahel Flisch, Stephanie Tschupp, Tracey Platt \& Willibald Ruch. 2012. How does psychopathy relate to humor and laughter? Dispositions towards ridicule and being laughed at, the sense of humor, and psychopathic personality traits. International Journal of Law and Psychiatry 35. 263-268.

Proyer, René T., Christian F. Hempelmann \& Willibald Ruch. 2009. Were they really laughed at? That much? Gelotophobes and their history of perceived derisibility. Humor: International Journal of Humor Research 22(1-2). 213-231.

Proyer, René T., Lukas Meier, Tracey Platt \& Willibald Ruch. 2013. Dealing with laughter and ridicule in adolescence: Relations with bullying and emotional responses. Social Psychology of Education 16(3). 399-420.

Proyer, René T. \& Monica Neukom. In press. Ridicule and being laughed at in the family: Gelotophobia, gelotophilia, and katagelasticism in young children and their parents. International Journal of Psychology.

Proyer, René T., Monica Neukom, Tracey Platt \& Willibald Ruch. 2012. Assessing gelotophobia, gelotophilia, and katagelasticism in children: An initial study on how six to nine-year-olds 
deal with laughter and ridicule and how this relates to bullying and victimization. Child Indicators Research 4. 1-20.

Proyer, René T., Tracey Platt \& Willibald Ruch. 2010. Self-conscious emotions and ridicule: Shameful gelotophobes and guilt free katagelasticists. Personality and Individual Differences 49.54-58.

Proyer, René T. \& Willibald Ruch. 2009a. How virtuous are gelotophobes? Self- and Peerreported character strengths among those who fear being laughed at. Humor: International Journal of Humor Research 22(1-2). 145-163.

Proyer, René T. \& Willibald Ruch. 2009b. Intelligence and Gelotophobia: The relations of self-estimated and psychometrically measured intelligence to the fear of being laughed at. Humor: International Journal of Humor Research 22(1-2). 165-181.

Proyer, René T. \& Willibald Ruch. 2010. Dispositions towards ridicule and being laughed at: Current research on gelotophobia, gelotophilia, and katagelasticism. Introduction to the special issue. Psychological Test and Assessment Modeling 52. 49-59.

Proyer, René T., Willibald Ruch, Numan S. Ali, Hmoud S. Al-Olimat, Toshihiko Amemiya, Tamirie Andualem Adal, Sadia Aziz Ansari et al. 2009. Breaking ground in cross-cultural research on the fear of being laughed at (gelotophobia): A multi-national study involving 73 countries. Humor: International Journal of Humor Research 22(1-2). 253-279.

Proyer, René T., Willibald Ruch \& Guo-Hai Chen. 2012. Gelotophobia: Life satisfaction and happiness across cultures. Humor: International Journal of Humor Research 25(1). 23-40.

Radomska, Anna \& Joanna Tomczak. 2010. Gelotophobia, self-presentation styles, and psychological gender. Psychological Test and Assessment Modeling 52. 191-201.

Renner, Karl-Heinz \& Timo Heydasch. 2010. Performing humor: On the relations between self-presentation styles, gelotophobia, gelotophilia, and katagelasticism. Psychological Test and Assessment Modeling 52.171-190.

Rawlings, David, Tsu Ann Tham \& Jessica Milner Davis. 2010. Gelotophobia, personality and emotion ratings following emotion-inducing scenarios. Psychological Test and Assessment Modeling 52. 161-170.

Ruch, Willibald. 2004. Gelotophobia: A useful new concept? IPSR Spring 2004 Colloquium Series, Department of Psychology, University of California at Berkeley.

Ruch, Willibald. 2009. Fearing humor? Gelotophobia: The fear of being laughed at. Introduction and overview. Humor: International Journal of Humor Research 22(1-2). 1-25.

Ruch, Willibald, Olga Altfreder \& René T. Proyer. 2009. How do gelotophobes interpret laughter in ambiguous situations? An experimental validation of the concept. Humor: International Journal of Humor Research 22(1-2). 63-89.

Ruch, Willibald, Ursula Beermann \& René T. Proyer. 2009. Investigating the humor of gelotophobes: Does feeling ridiculous equal being humorless? Humor: International Journal of Humor Research 22(1-2). 111-143.

Ruch, Willibald, Claudia Harzer \& René T. Proyer. 2013. Three by five: Three dispositions towards ridicule and being laughed at meet the Big Five. Israeli Journal of Humor Research: An International Journal 2. 2-20.

Ruch, Willibald, René T. Proyer \& Larry Ventis. 2010. The relationship of teasing in childhood to the expression of gelotophobia in adults. Psychological Test and Assessment Modeling 52. 77-93.

Ruch, Willibald \& René T. Proyer. 2008a. The fear of being laughed at: Individual and group differences in Gelotophobia. Humor. International Journal of Humor Research 21. 47-67. 
Ruch, Willibald \& René T. Proyer. 2008b. Who is gelotophobic? Assessment criteria for the fear of being laughed at. Swiss Journal of Psychology 67. 19-27.

Ruch, Willibald \& René T. Proyer. 2009a. Extending the study of gelotophobia: On gelotophiles and katagelasticists. Humor: International Journal of Humor Research 22(1-2). 183-212.

Ruch, Willibald \& René T. Proyer. 2009b. Who fears being laughed at? The location of gelotophobia in the PEN-model of personality. Personality and Individual Differences 46. 627-630.

Samson, Andrea C., Oswald Huber \& Willibald Ruch. 2010. Teasing, ridiculing and the relation to the fear of being laughed at in individuals with Asperger's Syndrome. Journal of Autism and Developmental Disorders 41. 475-483.

Samson, Andrea C. \& Yonni Meyer. 2010. Perception of aggressive humor in relation to gelotophobia, gelotophilia, and katagelasticism. Psychological Test and Assessment Modeling 52. 217-230.

Samson, Andrea C., René T. Proyer, Grazia Ceschi, Pier P. Pedrini \& Willibald Ruch. 2011. The fear of being laughed at in Switzerland: Are there regional differences and the role of positive psychology. Swiss Journal of Psychology 70(2). 53-62.

Samson, Andrea C., Pierre Thibault, René T. Proyer \& Willibald Ruch. 2010. The subjective assessment of the fear of being laughed at (gelotophobia): Adaptation of the French version of the GELOPH<15〉. European Review of Applied Psychology 60. 247-253.

Sarid, Orly, Willibald Ruch \& René T. Proyer. 2011. Gelotophobia in Israel: On the assessment of the fear of being laughed at. Israel Journal of Psychiatry and Related Sciences 48. 12-18.

Stefanenko, Ekaterina A., Alyona Ivanova, Sergey N. Enikopolov, René T. Proyer \& Willibald Ruch. 2011. The fear of being laughed at diagnostics: Russian adaption of gelotophobia questionnaire. Psychological Journal 32. 94-108.

Storch, Eric A., Deborah A. Roth, Meredith E. Coles, Richard G. Heimberg, Erica A. Bravata, \& Jason Moser. 2004. The measurement and impact of childhood teasing in a sample of young adults. Anxiety Disorders 18. 681-694.

Titze, Michael. 2009. Gelotophobia: The fear of being laughed at. Humor: International Journal of Humor Research 22(1-2). 27-48.

Ujlaky, Judit, René T. Proyer \& Willibald Ruch. 2012. The fear of being laughed at in Hungary: Assessing gelotophobia with the Hungarian version of the GELOPH<15>. In A.T. Litovkina, J. Sollosy, P. Medgyes \& W. Chlopicki (Eds.), Hungarian Humor, 271-283. Krakow, Poland: Tertium.

Weibel, Yves S. \& René T. Proyer. 2012. How do adults cope with laughter and ridicule? On the role of memories of teachers, family and peers. Psychologie in Erziehung und Unterricht 59. 81-92.

Weiss, Elisabeth M., Günter Schulter, Harald H. Freudenthaler, Ellen Hofer, Natascha Pichler \& Ilona Papousek. 2012. Potential markers of aggressive behavior: The fear of other persons' laughter and its overlaps with mental disorders. PLoS One 7. 


\section{Bionotes}

Willibald Ruch is a Full Professor of Psychology at the University of Zurich, Switzerland. His research interests are in the field of humor and laughter, cheerfulness and smiling. In his doctoral dissertation at the University of Graz, Austria in 1980 he developed a taxonomy of jokes and cartoons and its relation to personality. His more recent work, together with his research team at Zurich university, includes humor from a positive psychology perspective, the effectiveness of humor training programs and clown interventions, the ability to laugh at oneself, the fear of being laughed at (i.e., gelotophobia), and bringing gelotophobe-savvy laughter to avatars.

Jennifer Hofmann is a psychologist, doctoral student at the University of Zurich and member of the ISHS (International Society for Humor Studies). She is a human interaction expert working and lecturing in the field of humor.

Tracey Platt is a psychologist and project worker in the Department of Psychology at University of Zurich, Switzerland. She received her PhD from the University of Zurich in 2013. Her research interests are in human interaction, facial expression and emotion. She is the web-master to gelotophobia.org a website dedicated to gelotophobia assessment. Tracey has published a number of scientific journal articles on the subject of gelotophobia, as well as having co-authored a book chapter on the same topic. The International Society for Humor Studies, where she is a member, has also acknowledged her doctoral research by awarding her the 2010 Graduate Student Award.

René Proyer studied psychology (master level) at the University of Vienna (Austria). He received his $\mathrm{PhD}$ from the University of Zurich in 2006 and is currently a senior teaching and research associate at the Division of Personality and Assessment at the Department of Psychology at Zurich University. His main research interests are humor research (especially in the field of dispositions towards ridicule and being laughed at and adult playfulness), positive psychology (especially positive interventions), and test development. 
Bereitgestellt von | UZH Hauptbibliothek / Zentralbibliothek Zürich Angemeldet | 130.60.47.86

Heruntergeladen am | 16.12.13 10:07 